\title{
¿Cómo evaluar a estudiantes y residentes? Jornada de la Sociedad de Educación Médica de Euskadi (SEMDE)
}

Bilbao, 13 de diciembre de 2011

1.

\section{El video como herramienta para mejorar las habilidades docentes. Una experiencia con residentes de medicina de familia para valorar estas competencias}

Gil de Gómez-Barragán MJ, VázquezMedrano A, Ramón-Trapero JL

Unidad Docente de Medicina Familiar y Comunitaria. Servicio Riojano de Salud. Logroño, La Rioja.

Antecedentes. La grabación con video de las clases impartidas es un recurso educativo muy potente para el aprendizaje y la adquisición de competencias docentes y de comunicación. Objetivo. Mejorar las habilidades docentes y comunicativas de los residentes de medicina de familia utilizando una metodología basada en la experiencia, el ensayo con grabación y el feedback. Metodología. Experiencia educativa dirigida a los residentes de medicina de familia de primer año de La Rioja. El programa consistió en la realización inicial de un curso teóricopráctico con trabajos en grupos y role playing sobre una sesión docente. Posteriormente se programaron sesiones mensuales donde cada residente comunicaba un caso clínico. Esta sesión se grababa y posteriormente se visualizaba y analizaba con el fin de detectar áreas y compromisos de mejora. Los métodos de aprendizaje fueron: autoaprendizaje (lectura de artículos, trabajo personal de preparación de la sesión, análisis de sesiones y evaluación de docentes), videograbación, metodología aplicada (eva- luación de las habilidades docentes con una guía ad hoc), feedback individual (reproducción de la sesión grabada y entrevista individual con valoración y detección de aspectos y compromisos de mejora). Conclusiones. Las competencias docentes y de comunicación necesarias para preparar e impartir una sesión docente se adquieren mejor con una metodología basada en la experiencia y el ensayo. En nuestra unidad, la grabación con video de la exposición realizada por el residente y su posterior análisis con feedback grupal e individual ha mostrado ser una herramienta muy útil para valorar objetivamente estas competencias. La percepción de los residentes con esta experiencia es muy satisfactoria.

\section{2.}

Valoración de las encuestas de satisfacción a los padres de niños hospitalizados para detectar las necesidades de formación en comunicación de los pediatras

Astigarraga-Aguirre la, Latorre-Guisasola $\mathrm{M}^{\mathrm{b}}$

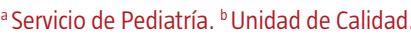
Hospital Universitario de Cruces. Barakaldo, Bizkaia.

Antecedentes. La formación sobre herramientas de comunicación del personal sanitario es fundamental para mejorar la seguridad y la satisfacción del paciente. Ciertas capacidades, como la empatía, la asertividad y la escucha activa no son innatas ni fruto de la experiencia, sino que deben po- tenciarse mediante el desarrollo de programas específicos. Objetivos. Analizar las encuestas de satisfacción realizadas a familiares para detectar problemas de trato, información y calidad percibida en la comunicación hospitalaria. Metodología. Revisión de encuestas de satisfacción realizadas a padres de niños hospitalizados en el Servicio de Pediatría del Hospital Universitario de Cruces en 2006, 2008 y 2010. Estudiar las preguntas y respuestas relacionadas con información y trato del personal sanitario, peculiaridades en la infancia y tendencia en la percepción de los padres. Resultados. Se realizaron 755 entrevistas telefónicas individuales válidas a padres de niños ingresados, con un mismo cuestionario de 56 preguntas. Las encuestas incluyeron 10 preguntas relacionadas con trato y empatía (nueve referidas a médicos o enfermeras) y 12 cuestiones sobre información proporcionada por profesionales sanitarios. La satisfacción fue buena, muy buena o excelente ( $>70 \%$ ) en 20 respuestas analizadas, la tendencia fue favorable y se alcanzaron los objetivos previstos de accesibilidad, información y trato al usuario/paciente en la mayoría. Un aspecto a mejorar es la información directa sobre la enfermedad a los niños (55-60\% contestan 'sí). Conclusiones. La mayoría de los padres de niños hospitalizados están satisfechos con el trato, empatía e información recibida de médicos y enfermeras. La formación en habilidades de comunicación es importante para mejorar la calidad percibida por los padres. Los pediatras precisan educación específica para tratar con niños y adolescentes.
3.

Seguimiento y evaluación del proceso de enseñanzaaprendizaje en un programa formativo basado en metodologías activas de la asignatura 'Farmacología clínica' de quinto curso de medicina

Pineda J, Mendiguren A

Departamento de Farmacología. Facultad de Medicina y Odontología. Universidad del País Vasco (UPV/EHU). Leioa, Bizkaia.

Antecedentes. En 2009-2010 se diseñó e implementó un programa formativo orientado al uso de metodologías activas tipo ABP (aprendizaje basado en problemas) en la asignatura 'Farmacología clínica' de quinto de medicina. Objetivos. Hacer un seguimiento y evaluación del proceso de enseñanza-aprendizaje de esta asignatura en 2010-2011 tras incorporar en el diseño $A B P$ algunas mejoras que se identificaron previamente. Metodología. Implementamos la experiencia ABP de 'Farmacología clínica' en los grupos de Basurto y Donostia y reevaluamos los resultados mediante análisis evolutivo del rendimiento académico y nivel de satisfacción del alumnado, análisis de las competencias iniciales y adquiridas en el proceso, y valoración global, comparativa y evolutiva de la opinión del alumnado sobre el ABP. Resultados. El diseño del programa de la asignatura incorporó tres tipos de mejoras: mayor concreción del sistema evaluativo, gestión más ajustada del tiempo de 
las tareas y presentación de una guía detallada de la actividad ABP. Los 65 estudiantes que realizaron la experiencia alcanzaron una nota media de $7,9 \pm 0,2$ puntos. El rendimiento académico de la muestra mejoró respecto al curso 2009-2010, con un 10$20 \%$ más de estudiantes con notas elevadas. A comienzo de curso, el alumnado valoró que tenía una alta capacitación en las competencias transversales, pero insuficiente en las competencias específicas de la asignatura. Al finalizar la asignatura, el sistema evaluativo confirmó la alta calificación de las competencias transversales; las competencias específicas de razonamiento clínico y toma de decisiones fueron muy bien calificadas, pero las competencias más profesionalizadoras tuvieron peores notas (notable bajo). La opinión del alumnado sobre el ABP se mantuvo alta para la mayoría de los descriptores y mejoró en uno (número de sesiones). Más del $85 \%$ consideró que con el $A B P$ se aprendía más que con otras metodologías y que había una relación más integrada con la práctica médica. La opinión general del alumnado se mantuvo por encima de 4 (sobre 5). Conclusiones. Todos los indicadores empleados para medir el aprendizaje de competencias, la motivación y el rendimiento académico son muy favorables con esta experiencia ABP. Algunos estudiantes muestran, no obstante, una actitud ambigua sobre el sobreesfuerzo realizado.

4.

Iniciación a la realidad médica. El cine como recurso en la enseñanza-aprendizaje de habilidades médicas

Ortiz-Jauregi MA, González-Pinto Arrillaga A

Departamento de Neurociencias. Facultad de Medicina y Odontología. Universidad del País Vasco (UPV/EHU). Leioa, Bizkaia.

Antecedentes. La adaptación al Espacio Europeo de Educación Superior ha estimulado el desarrollo de nuevas estrategias de enseñanza-aprendizaje, donde los profesores de las áreas de salud hemos sido testigos y acto- res de un nuevo paradigma de enseñanza-aprendizaje basado en competencias necesarias para el ejercicio de la profesión médica en su totalidad. Objetivos. Diseño de acciones de innovación en la enseñanza-aprendizaje de habilidades médicas psicológicas en los estudiantes de medicina mediante la elaboración de material didáctico a través del uso del cine. Metodología. Se seleccionaron 5-6 películas comerciales donde era posible analizar aspectos específicos de la práctica médica o de la psicología médica y psiquiatría. Posteriormente, se procedió a la selección de secuencias ilustrativas de los diferentes aspectos de la relación médico-paciente y al análisis de ellas. Los estudiantes de primer curso del Grado de Medicina vieron las películas y debatieron sobre las viñetas seleccionadas en el pequeño grupo y sesiones plenarias. Resultados. Los estudiantes valoran muy positivamente la actividad. La metodología posibilita el acceso y la consideración de la relación médico-paciente y de las vivencias de los médicos, en su actividad profesional, y de los pacientes, en su enfermedad, aspectos cuya vivencia sería muy difícil experimentar. Conclusiones. Consideramos este proyecto capaz de promover el aprendizaje activo de los estudiantes con un enfoque innovador y proactivo, basado en el uso de un vehículo cercano al estudiante como base para las discusiones de grupo y la comprensión de conductas y actitudes, así como para el modelado de éstas.

\section{5.}

Competencias adquiridas durante la formación de grado y al final de la formación especializada: análisis de las relacionadas con valores profesionales, actitudes, comportamiento y ética

Morán-Barrios Ja , Martínez-Indart La , Téllez $\mathrm{R}^{\mathrm{a}}$, González-Larragán $\mathrm{S}^{\mathrm{a}}$, González-García MJ a, Cerqueira MJ b, Martínez-Landeras V ${ }^{c}$, Ortubai Ad, Rodríguez Je, Telletxea $S^{f}$, Villalonga R ${ }^{g}$

a Unidad de Docencia Médica. Hospital de Cruces. Barakaldo, Bizkaia. ${ }^{b}$ Hospital Vall d'Hebron. Barcelona. ' Hospital Marqués de Valdecilla. Santander. ${ }^{d}$ Unidad de Medicina Familiar y Comunitaria de Álava. ${ }^{e}$ Hospital Central de Asturias. Oviedo. ${ }^{\mathrm{f}} \mathrm{Hospital}$ de Galdakao. ${ }^{9} \mathrm{Hospital}$ de Bellvitge. Barcelona.

Introducción. Gran parte de las actitudes y comportamientos de los futuros profesionales se adquieren en los procesos de formación y condicionan los estilos de práctica y ejercicio profesional, lo que determina la relación de la profesión médica con la sociedad, la tecnología y otros agentes sanitarios. Objetivos. Conocer las competencias adquiridas relacionadas con los valores profesionales, actitudes, comportamiento y ética -modelo de competencias (Global Minimum Essential Requirements in Medical Education)- en dos colectivos: licenciados procedentes de un grupo de universidades españolas que iniciaron su especialización en siete hospitales o unidades docentes españolas en el año 2010 ( $n=330$ ) y residentes al final de su especialización en el Hospital de Cruces, entre 2009 y 2011 ( $n=139)$. Metodología. Autoevaluación mediante un formulario de 54 ítems (tasa de respuestas: 91,5\%; $\alpha$ de Cronbach = $0,942)$. Escala de valoración: 0, na$\mathrm{da} /$ muy deficiente; 1 , poco/deficiente; 2 , adecuado/bien; 3, mucho/muy bien. Análisis: descriptivo. Resultados. a) Principios éticos, responsabilidades legales de la profesión médica y el secreto profesional para el beneficio del paciente, de la sociedad y la profesión: inicio residencia, 2,24 \pm 0,66; final residencia, 2,49 $\pm 0,58$. b) Saber aplicar el principio de justicia social a la practica profesional: inicio residencia, 1,97 $\pm 0,70$; final residencia, 2,35 $\pm 0,57$. c) Respetar la autonomía del paciente, sus creencias y cultura: inicio residencia, 2,40 $\pm 0,60$; final residencia, 2,59 \pm 0,50. d) Reconocer las propias limitaciones y la necesidad de mantener y actualizar su competencia profesional: inicio residencia, 2,31 \pm 0,61 ; final residencia, 2,64 $\pm 0,48$. e) Desarrollar la práctica profesional con respeto a otros profesionales de la salud: inicio residencia, 2,32 $\pm 0,70)$; final residencia, 2,62 $\pm 0,50$. Conclusiones. La percepción de formación en determinados valores profesionales en el periodo universitario no es deficitaria (media $\geq 2$ ), estando en el límite la aplicación del principio de justicia. Al final de la especialización mejoran todos los ítems, aunque ambos colectivos no son comparables.

6.

Nuevas herramientas para el tutor: Google Documents ${ }^{\circledR}$

Saá $\mathrm{R}^{\mathrm{a}}$, Colina $\mathrm{A}^{\mathrm{b}}$

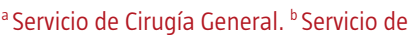
Aparato Digestivo. Hospital Universitario de Cruces. Barakaldo, Bizkaia.

Antecedentes. Desde los años noventa, la formación de posgrado en el ámbito anglosajón cuenta con plataformas virtuales. La aparición en Estados Unidos de los Journal Clubs permitía a los residentes participar en actividades docentes fuera del horario laboral una vez que este había quedado reducido a 80 horas semanales. Existen múltiples herramientas informáticas que se han utilizado para intentar cumplir las exigencias planteadas por el Accreditation Council for Graduate Medical Education (ACGME). Web CT Vista ${ }^{\circledR}$, Macromedia Breeze ${ }^{\circledR}$, MedFiler ${ }^{\circledR}$ y otros programas son ejemplos de cómo se ha intentado optimizar la formación de posgrado sin mermar su calidad. Casi todas ellas son herramientas de pago o presentan restricciones de uso en el medio hospitalario porque precisan instalación y asistencia técnica para su utilización. Objetivos. Conocer el estado real de la docencia de posgrado en nuestro medio, detectar las deficiencias del sistema y aplicar medidas de mejora, implementar Google Documents ${ }^{\circledR}$ como sistema universal de gestión de la tutoría, aumentar la exigencia y la calidad, organizar eventos científicos propios, gestionar una vía de publicación e innovar en alternativas para el entrenamiento quirúrgico. Metodología. Para tener una visión más objetiva de la docencia de posgrado en nuestro entorno realizamos una encuesta de 14 preguntas a 23 residentes del País Vasco, sobre aspectos relacionados con la calidad de la enseñanza y con elementos indicativos de su relación con plataformas virtuales y redes sociales. Escogimos 
Google Documents por ser una aplicación que permitía tanto recabar información como aportar la que creyésemos oportuna para su formación. Nos planteamos la búsqueda de un método para estimular las publicaciones y un foro propio de debate científico. Resultados. Como elementos más destacados de la encuesta detectamos que el $43 \%$ no reconoce seguimiento alguno de su formación; se apoyan en los residentes mayores para escoger la bibliografía de la especialidad. El 83\% plantea que nadie le ayuda a publicar y tiene que utilizar sus propios recursos. Diecinueve encuestados (83\%) no sigue ningún calendario de temas para su estudio individual y la totalidad prefiere evaluaciones periódicas en vez de una evaluación final. Una gran mayoría (83\%) coincide en que sería más útil mantener informado periódicamente al tutor de su actividad práctica contra una minoría que lo haría en la memoria final. El $61 \%$ utiliza de forma más o menos frecuente las redes sociales, y a todos les ha parecido interesante contar con una plataforma donde consultar bibliografía, calendarios y otros aspectos de su interés. Creamos un sistema de carpetas en Google Docs donde les aportábamos: un calendario fijo de rotaciones y actividades docentes propias; por año de residencia, artículos resumen revisados y descargados por nosotros de revistas de alto impacto para asegurar un mínimo de material de estudio, vídeos de técnicas quirúrgicas básicas, revisiones de técnicas avanzadas, una base de datos donde ir incorporando su actividad quirúrgica al día, un foro de mensajes, dudas, quejas y sugerencias, resultados de evaluaciones periódicas tanto prácticas como teóricas y resultados de las encuestas de satisfacción periódicas. Pactamos con una editorial de publicaciones científicas por Internet (QuickMedPub) el envío frecuente de artículos de investigación o casos de especial interés. Organizamos anualmente una Jornada de Casos Clínicos para los residentes de la CAV en la que ellos comienzan a ser parte del jurado. Creamos un sistema permanente de entrenamiento básico en cirugía convencional y laparoscópica utilizando en gran parte ma- terial fabricado por nosotros. Hemos aplicado y automatizado un sistema de evaluación práctica por objetivos (OSATS) en cada rotación. Realizamos también la evaluación por competencias de forma online con los diferentes responsables de sus rotaciones. Incluimos una estancia externa en su calendario de formación pactada previamente. Conclusiones. La formación de posgrado en nuestro medio necesita un impulso especial para alcanzar estándares aceptables de calidad. Google Documents se ha revelado como una excelente herramienta para optimizar los esfuerzos en la formación del residente al aportar una gran capacidad de gestión. Si la implicación del tutor es alta, se pueden conseguir buenos resultados porque existen muchos recursos docentes infrautilizados. La gratuidad de esta aplicación es otro atractivo añadido y por eso recomendamos su utilización.

\section{7.}

Portafolio reflexivo basado

en competencias. Un método

de evaluación formativa

del proyecto 'Formación especializada basada en competencias' en el Hospital de Cruces (2008)

Morán-Barrios J, Somme J, Téllez R, Iglesias G, Sagastagoya J, Amutio E, Basterretxea A, Bereziartua E, Iriberri M, Laita A, Martínez-Berriotxoa A, Rodríguez-Iñigo MA, González-Larragán S, González-García MJ

Hospital de Cruces. Barakaldo, Bizkaia.

Antecedentes. El aprendizaje de adultos se basa en la 'acción-reflexión'. La reflexión del residente sobre las actividades realizadas y su documentación forman la parte más fundamental del aprendizaje. Objetivos. Introducir la formación especializada basada en competencias (FEBC) en un hospital de tercer nivel (300 residentes, 40 especialidades), a través de métodos de evaluación del residente formulados por competencias, siendo uno de ellos el portafolio reflexivo basado en competencias (PRBC). Metodología. 1) Año 2008: La Unidad de
Docencia Médica inicia el proyecto definiendo un marco de referencia basado en siete dominios competenciales ('visión Docente': profesionalidad, cuidados del paciente, comunicación, conocimientos, práctica basada en el contexto del sistema de salud, manejo de la información y práctica basada en el aprendizaje y la mejora continua. 2) Inclusión en el plan estratégico. 3) Desarrollo a través de una masa crítica de tutores y residentes. 4) Diseño de un sistema de evaluación formativa basado en siete dominios competenciales: nuevos formularios de evaluación formativa y autoevaluación. 5) PRBC sobre la base del documento diseñado en 1999: incluye reflexiones libres estructuradas en cada uno de los dominios referidos, sobre la formación adquirida en cada periodo o rotación, además de aspectos cuantitativos. Resultados. Desarrollo del PRBC en 2010 y 2011: de 537 documentos, el $85 \%$ incluyen reflexiones por competencias. Aplicación de los formularios de evaluación formativa y entrevista tutor-residente (autoevaluación). Conclusiones. El PRBC desarrolla las siguientes competencias: autoaprendizaje reflexivo, comunicar por escrito los aprendizajes, rendir cuentas a la institución y ayudar a introducir la FEBC. Es posible poner en práctica la FEBC en residentes sin experiencia previa en sus universidades. Según nuestro conocimiento, ésta es la primera experiencia de FEBC en hospitales españoles.

8.

Método de caso: resultado en el aprendizaje y la evaluación

Ruiz de Alegría B, De Lorenzo E, Basurto S, Ulibarri A.

Escuela Universitaria de Enfermería. Vitoria-Gasteiz.

Introducción. Las directivas legislativas de los estudios de educación superior exigen nuevos enfoques pedagógicos fomentando metodologías activas. El método de caso es una metodología activa centrada en desarrollar las competencias que permitan la incorporación eficaz al ámbito laboral. Objetivos. Conocer la valoración que hacen los estudiantes de enfermería del método de caso, su contribución al desarrollo de competencias y el nivel de satisfacción con el aprendizaje. Metodología. Se utilizó un cuestionario elaborado ad hoc, una vez confrontada su validez con expertos. Se pasó a 77 estudiantes de primero de enfermería de la Escuela Universitaria de Enfermería de Vitoria-Gasteiz después de realizar una intervención pedagógica con el método de caso, que consistió en exponer al estudiante a una toma de decisiones, partiendo de una situación real en la que el contexto era un factor modulador. Resultados. El 88\% expresaron tener más interés y motivación por la asignatura. Considerando sólo las respuestas 'bastante' o 'mucho', el 100\% manifestaron que les ayudó a establecer relación entre teoría y práctica; el $96 \%$, que les ayudó a avanzar en su desarrollo competencial; el 93\%, que les ayudó en la toma de decisiones, y el $96 \%$, que les ayudó a analizar situaciones de la práctica profesional. Conclusiones. El método de caso permite evaluar las competencias de: resolución de problemas, toma de decisiones, análisis crítico, argumentación, comunicación oral y escrita y razonamiento ético. El estudiante valora con un alto grado de satisfacción el alcance de las competencias diseñadas y reconoce que este método ayuda a mejorar la relación entre teoría y práctica y a enlazar los diferentes conocimientos. 